\title{
A monolithic single-chip point-of-care platform for metabolomic prostate cancer detection
}

\author{
Valerio F. Annese $\mathbb{E D}^{1}$, Samadhan B. Patil ${ }^{1}$, Chunxiao Hu${ }^{1}$, Christos Giagkoulovits ${ }^{1}$, Mohammed A. Al-Rawhani ${ }^{1}$, \\ James Grant ${ }^{1}$, Martin Macleod², David J. Clayton³ , Liam M. Heaney $\mathbb{D}^{4}$, Ronan Daly ${ }^{5}$, Claudio Accarino ${ }^{1}$, Yash D. Shah', \\ Boon C. Cheah', James Beeley ${ }^{1}$, Thomas R. Jeffry Evans ${ }^{6}$, Robert Jones ${ }^{6}$, Michael P. Barrett ${ }^{5,7}$ and David R. S. Cumming ${ }^{1}$
}

\begin{abstract}
There is a global unmet need for rapid and cost-effective prognostic and diagnostic tools that can be used at the bedside or in the doctor's office to reduce the impact of serious disease. Many cancers are diagnosed late, leading to costly treatment and reduced life expectancy. With prostate cancer, the absence of a reliable test has inhibited the adoption of screening programs. We report a microelectronic point-of-care metabolite biomarker measurement platform and use it for prostate cancer detection. The platform, using an array of photodetectors configured to operate with targeted, multiplexed, colorimetric assays confined in monolithically integrated passive microfluidic channels, completes a combined assay of 4 metabolites in a drop of human plasma in under $2 \mathrm{~min}$. A preliminary clinical study using L-amino acids, glutamate, choline, and sarcosine was used to train a cross-validated random forest algorithm. The system demonstrated sensitivity to prostate cancer of $94 \%$ with a specificity of $70 \%$ and an area under the curve of 0.78 . The technology can implement many similar assay panels and hence has the potential to revolutionize low-cost, rapid, point-of-care testing.
\end{abstract}

\section{Introduction}

One in two people will develop cancer at some point in their lifetime ${ }^{1}$. The World Health Organization estimates that cancer is the second leading cause of death globally, claiming 9.6 million lives in 2018 alone $^{2}$. Cancer incidence is expected to rise by more than $40 \%$ in the next 15 years as the population ages ${ }^{3-5}$. With an estimated 1.3 million cases and 0.4 million deaths worldwide in 2018, prostate cancer ( $\mathrm{PCa}$ ) is the most commonly diagnosed cancer in developed countries and the sixth leading cause of cancer deaths in $\operatorname{men}^{5-7}$. Although the frequency and survival rate vary considerably for $\mathrm{PCa}$, there is consistent evidence that patients diagnosed at an early stage are more likely to survive ${ }^{2,3}$. Early diagnosis makes localized treatments, including prostatectomy and radiotherapy,

\footnotetext{
Correspondence: Valerio F. Annese (valerio.annese@glasgow.ac.uk) ${ }^{1}$ Electronics and Nanoscale Engineering, James Watt School of Engineering, University of Glasgow, Glasgow G12 8QQ, UK

2Beatson West of Scotland Cancer Centre, Glasgow G12 OYN, UK

Full list of author information is available at the end of the article
}

possible; hence, the 5 -year survival rate is nearly $100 \%^{3,8}$. Nevertheless, the survival rate drops to $34 \%$ when a tumor is diagnosed in a late metastatic stage ${ }^{8}$.

Despite this, a robust and effective PCa screening program is not available today ${ }^{9,10}$. The widely used prostatespecific antigen (PSA) test, which is the current standard blood test for PCa diagnosis, has been found to be unreliable; fewer than one in three men with an elevated PSA will have $\mathrm{PCa}^{11}$, and the test misses $\sim 15 \%$ of tumors $^{12}$. The high false-positive rate of the PSA test can lead to unnecessary medical procedures such as digital rectal examination, MRI, and biopsy. In addition to being painful, invasive, and having the potential to cause complications, PSA downstream tests can be expensive ${ }^{13}$, accounting for more than $70 \%$ of the medical costs associated with PCa screening. While the use of the PSA test alone is problematic ${ }^{14}$, it has also been suggested that PSA could still be a valuable complement to new and emerging tests such as the one we propose ${ }^{15}$.

\section{(c) The Author(s) 2021}

(c) (i) Open Access This article is licensed under a Creative Commons Attribution 4.0 International License, which permits use, sharing, adaptation, distribution and reproduction cc) in any medium or format, as long as you give appropriate credit to the original author(s) and the source, provide a link to the Creative Commons license, and indicate if changes were made. The images or other third party material in this article are included in the article's Creative Commons license, unless indicated otherwise in a credit line to the material. If material is not included in the article's Creative Commons license and your intended use is not permitted by statutory regulation or exceeds the permitted use, you will need to obtain permission directly from the copyright holder. To view a copy of this license, visit http://creativecommons.org/licenses/by/4.0/. 
While numerous microtechnologies have been proposed to improve and miniaturize PSA tests ${ }^{16-18}$, an independent alternative is sought. One such alternative is to use a panel of metabolite markers that, when taken together, can be analyzed to yield a sensitive and specific test ${ }^{19-27}$. The creation of a panel-based test requires a technological platform that is capable of making multiple simultaneous measurements, ideally in a point-of-care (POC) format that lends itself to regular screening and monitoring that has been shown to be beneficial ${ }^{28}$. Here, we propose the use of a microelectronic test platform based on complementary metal-oxide silicon (CMOS) that underpins all integrated circuit technology. CMOS has the potential to revolutionize multimetabolite marker panel measurements for many diseases, including PCa. Chips with integrated sensors and readouts have been successfully used for single measurements, such as glu$\operatorname{cose}^{29}$, targeted DNA sequences ${ }^{30}$ and intracellular transmembrane potentials ${ }^{31}$; multiple identical measurements, e.g., genome sequencing ${ }^{32}$; and multianalyte measurements using partitions over a sensing area ${ }^{33}$.

Studies to make devices using micromachining ${ }^{34}$, additive manufacturing ${ }^{35}$, and replica molding ${ }^{36,37}$ have been carried out, but to date, none of these has proven capable of meeting the multimetabolite measurement challenge that must be addressed to build a POC marker panel system. Current methods for CMOS/microfluidic integration and packaging are complex and costly ${ }^{38}$. In addition to building a physical device architecture, solutions are also required for microchannel functionalization, reagent stability ${ }^{39}$, and minimizing crosstalk $^{40}$. Finally, these systems should work with minimal sample preprocessing 39 .

We have overcome these barriers by monolithically integrating a passive microfluidic system onto a CMOS sensor chip to measure multiple metabolites directly from a single droplet of plasma. To do this, it was necessary to control the material dimensions to ensure consistent optical measurements were possible, to control the surface chemistry, hence hydrophilicity, of the channels to ensure passive sampling occurred, and to introduce multiple channel biochemical functionalization on the same chip. In this work, we focused on PCa to demonstrate a new technology with the capability for wideranging application and impact.

As described in the Supplementary Information, we selected a panel of 4 metabolites made up of total $\mathrm{L}$-amino acids (LAA), glutamate, choline, and sarcosine. After calibration, the platform was used in preliminary clinical trials with human plasma from 10 healthy men and 16 men diagnosed with PCa. Metabolite profiles were used to train a random forest classifier algorithm. The classifier was shown to have a cross-validated sensitivity of $94 \%$ and a specificity of $70 \%$ when discriminating between samples from patients with and without $\mathrm{PCa}$, improving upon the current PSA-based clinical standard in the population that we studied.

\section{The platform}

The POC platform is made up of three units (Fig. 1): the disposable chip cartridge, the reader, and the GUI. The apparatus performs colorimetric quantification of a chosen metabolite panel.

\section{Metabolite panel for PCa}

Cancer cells experience rapid proliferation, and their metabolism diverges from healthy cells, giving rise to changes that can be reflected in global measures of the human metabolome ${ }^{41-43}$. Cancer-related metabolites accumulate in human body fluids, and their levels can act as indicators or biomarkers to identify or monitor the disease $^{44,45}$. Many blood metabolites have been found to
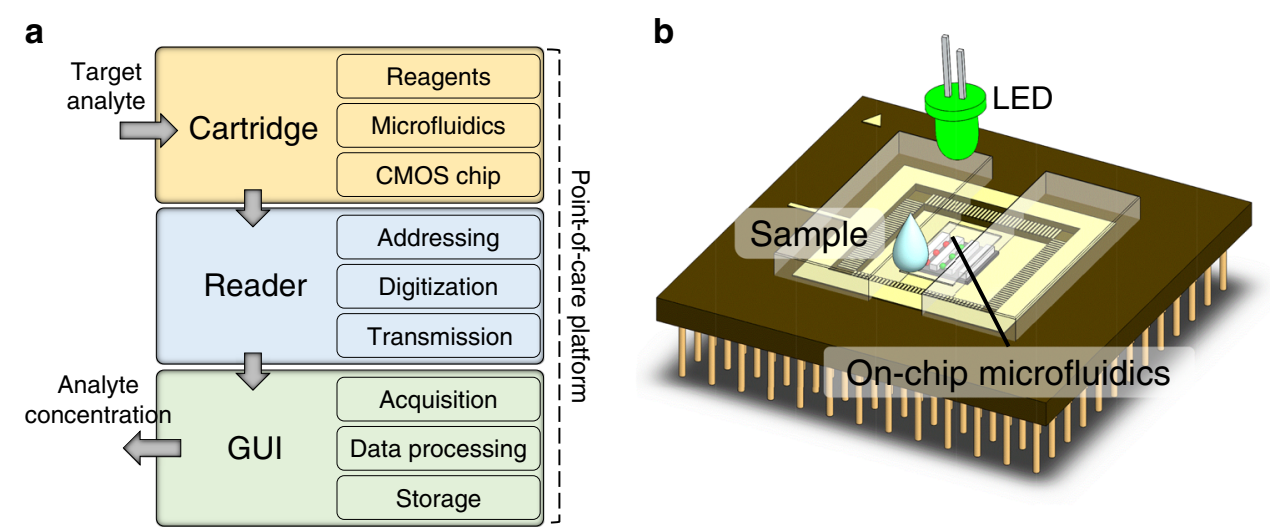

Fig. 1 Platform architecture and cartridge. a Schematic architecture of the platform showing the cartridge that needs only a drop of sample to perform a measurement, the reader, and the computing device for use as a GUI. b A sketch of the multiple measurement cartridge device used in this work with a CMOS chip, passive microfluidics, a chip package, and an optical assembly. 

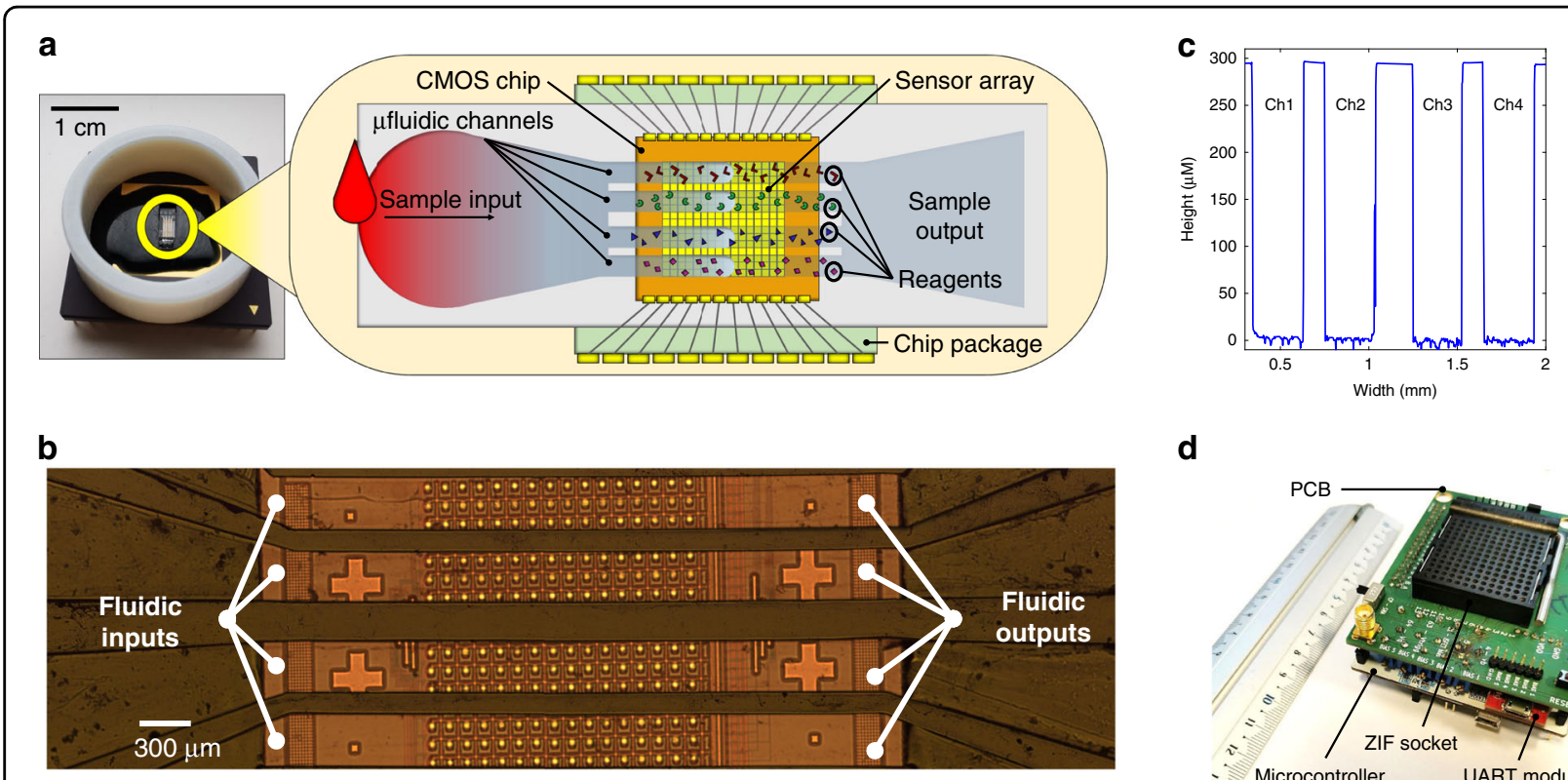

d

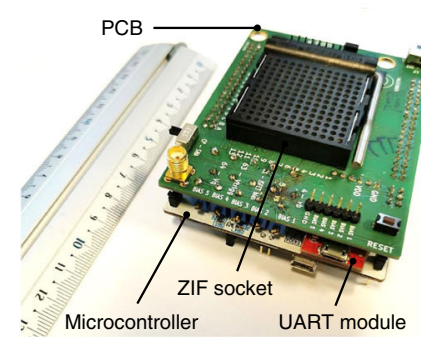

Fig. 2 Cartridge and reader. a The cartridge (left) and schematic diagram of its main components (right). $\mathbf{b}$ Micrograph of the microfluidics fabricated on the chip's sensitive area. c Profile of the microstructure built on the sensitive area measured with a Bruker Contour GT-X 3D Optical Profiler (d). The handheld reader removed from its enclosure.

be linked to $\mathrm{PCa}^{19-27,44-48}$. Among them, the progression of cancer is associated with the modification of specific transporters, namely, large amino acid transporters 1 and 3 (LAT1 and LAT3) $)^{19}$, which can yield an alteration of the blood LAA profile ${ }^{20,23,24,46,47}$. Cancer cells have been shown to have an upregulated glutamine-glutamate energy cycle; hence, glutamate represents an excellent candidate marker for $\mathrm{PCa}^{20,47,48}$. The modification of choline levels, arising from alterations of the enzyme choline kinase- $\alpha$ and the CHT1 choline transporter, has been detected in connection with $\mathrm{PCa}^{21,22}$. Finally, sarcosine has also been linked, albeit variably, to $\mathrm{PCa}$ for diagnosis $^{24}$, malignancy assessment ${ }^{23-25}$, and staging $23,24,26,27$. While the evidence that sarcosine is a useful marker for $\mathrm{PCa}$ is debated ${ }^{49,50}$, we elected to add it to the present study. A more detailed review of metabolomics for PCa and our panel selection is provided in the Supplementary Information.

\section{Detection strategy}

The platform used in this study was developed to quantify the aforementioned candidate metabolic biomarkers using colorimetry. Biological reagents were selected to produce a measurable light absorbance change at a specific wavelength after the interaction with the target metabolite. The initial rate of the reaction is linked to the initial concentration of the metabolite by the Michaelis-Menten model ${ }^{51}$. For the colorimetric determination of LAA, glutamate, choline, and sarcosine, a two-stage reaction process was used to conduct measurements. In the first reaction step, a substratespecific enzyme was used to produce $\mathrm{H}_{2} \mathrm{O}_{2}$; we used LAA oxidase (LAAOX E.C.-1.4.3.2), glutamate oxidase (GLOX E.C.-1.4.3.11), choline oxidase (CHOX E.C.-1.1.3.17), and sarcosine oxidase (SAOX E.C.-1.5.3.1). The $\mathrm{H}_{2} \mathrm{O}_{2}$ produced was in turn monitored by a colorimetric probe that changed its absorbance properties depending on the $\mathrm{H}_{2} \mathrm{O}_{2}$ concentration. Phenol and 4-aminoantipyrine (4-AAP) were used in this work. In the presence of the catalyzing enzyme peroxidase (HRP), phenol and 4-AAP react with $\mathrm{H}_{2} \mathrm{O}_{2}$, producing quinone imine, which has higher light absorbance in the range of $400-600 \mathrm{~nm}$. The absorbance is linked to the rate of the reaction by the Beer-Lambert law ${ }^{52}$. Detection of an electronic signal was performed using the platform's array of photodiodes (PDs) to measure the colorimetric reaction.

\section{The cartridge}

The cartridge was made using a ceramic 120 pin grid array (PGA) chip package, a custom complementary metal-oxide-semiconductor (CMOS) chip, a microfluidic capillary network fabricated directly on the chip, and biological reagents. A schematic representation of the main components embedded in the cartridge is shown in Fig. 2a.

The CMOS chip was fabricated using a commercially available 350-nm high-voltage 4-metal process provided by austriamicrosystems (AMS). The chip integrates a $16 \times$ 16 array, or frame, of multisensing elements. Each multisensing element comprises a PD, an ISFET, and a 
single-photon avalanche diode (SPAD) ${ }^{53,54}$. Only the PDs were used in this work. Each multisensing element is $100 \times 100 \mu \mathrm{m}$ in size, leading to a total sensitive area of $1.6 \times 1.6 \mathrm{~mm}$. The size of the entire CMOS chip is $3.4 \times$ $3.6 \mathrm{~mm}$. The CMOS chip was wire-bonded into the PGA.

On top of the sensing area, a passive microfluidic network was monolithically integrated, providing physical separation for parallel testing so that more than one metabolite could be measured at the same time. The walls of the microstructure were fabricated on top of the CMOS chip using a biocompatible black epoxy resin (302-3M 1LB by Epoxy Technology Inc.) using a combination of soft lithography and injection molding. A plain polydimethylsiloxane (PDMS) slab coated with polyvinyl alcohol (PVA $)^{55}$ was bonded onto the epoxy-based structure by plasma oxidation to enclose the channels from the top. A detailed description of the microfluidic integration is presented in the Materials and Methods section of this paper. As shown in Fig. 2b, c, the microchannel height, width, and length were $\sim 291.95 \pm$ $6.44 \mu \mathrm{m}, 300.87 \pm 0.86 \mu \mathrm{m}$ and $4.0 \pm 0.1 \mathrm{~mm}$, respectively. A liquid sample (see "Materials and methods" section) was introduced to the cartridge using a pipette (Finntip ${ }^{\mathrm{TM}}$ F2 by ThermoFisher). Once on the cartridge, the sample under test was divided into four identical microfluidic channels that physically confined the reactions in each channel.

Two types of experiments were conducted: a series to measure each metabolite one-by-one to assess the validity of the proposed panel and a second to make four measurements in parallel and demonstrate the potential of the platform. When testing a single metabolite, the sample and the bioreagent were mixed in the liquid state immediately before loading onto the chip to perform the test. Thus, both the sample and bioreagents are introduced to the system at the same time. When testing multiple metabolites in parallel, the channels were individually functionalized with the different bioreagents required to detect each metabolite. Biological reagents were preloaded into the microchannels by manual pipetting and then dried. The procedure entrapped and isolated the solid materials in their respective microchannels.

\section{Reader and graphical user interface}

The cartridge plugs into the reader using a zeroinsertion force (ZIF) socket. The reader is $8.5 \times 7.5 \times$ $4.0 \mathrm{~cm}$ and weighs $150 \mathrm{~g}$. The reader provides functionality for sensor multiplexing and addressing, data digitization and transmission to a personal computing device via a USB link. The reader is based around an STM32F334R8T6 microcontroller on an ST Nucleo F334R8 board (Fig. 2d) that is programmed before use with custom firmware. Data are digitized using the embedded 12-bit analog to digital converter with an average rate of 36 frames per second. The reader is powered by the USB link $(5 \mathrm{~V})$, which in turn powers the cartridge $(3.3 \mathrm{~V})$. The reader also has an LED $(\lambda=490 \mathrm{~nm}$, FWHB $=20 \mathrm{~nm}$ ). Using a lens (AC254-035-A-ML BBAR Coated, $f=35 \mathrm{~mm}$ lens from Thorlabs), the LED illuminates the sensing area of the cartridge with collimated light. The GUI, based on custom software and running on a personal laptop (HP EliteBook i7-8650u 16 GB), interfaces with the reader (via USB) and performs data acquisition, display, analysis, and storage. The results can also be uploaded onto a cloud. The GUI also performs offline signal processing. Additional details (Supplementary Fig. 4) of the GUI are reported in the Supplementary Information.

\section{Results}

The platform was tested and characterized using human plasma samples modified with known concentrations of metabolites so that calibration curves could be generated. Subsequently, a preliminary clinical study for $\mathrm{PCa}$ was performed using ten samples from healthy men not known to have PCa (non-PCa group) and sixteen samples from men affected by $\mathrm{PCa}$.

\section{Calibration}

Calibration curves for LAA, glutamate, choline, and sarcosine in human plasma are shown in Fig. 3. The complete characterization of the platform for the analytes of interest is presented in Table 1. At least six data points were used to obtain the calibration curves for each metabolite. Each data point was obtained as the average over three replicates (see "Materials and methods" section). Kinetic constants were estimated by fitting data to the Michaelis-Menten model. The $K_{\mathrm{m}}$ results for all of the metabolites were in line with the values reported in the literature ${ }^{56}$. For all the metabolites, the goodness of fit with the Michaelis-Menten model was satisfactory with $R^{2}$ values $\geq 0.97$. For substrate concentrations lower than $K_{\mathrm{m}}$, the data were also fitted using a linear model. For all the metabolites, high linearity was observed in the concentration range of interest $\left(R^{2} \geq 0.93\right)$. The linear ranges were in line with the physiological ranges. Typical standard deviations for the measurements in the linear range were found to be $16-20 \%$.

\section{Limit of detection and limit of quantification}

The limit of detection (LOD) and limit of quantification (LOQ) were quantified using the "International Union of Pure and Applied Chemistry" (IUPAC) definition ${ }^{57}$. The average $\left(\mu_{\mathrm{c}}\right)$ and standard deviation $\left(\delta_{\mathrm{c}}\right)$ of the initial reaction rate for negative controls (common to all the assays) were found to be 5 and $2.7 \mu \mathrm{V} \mathrm{s}^{-1}$, respectively. Consequently, the LOD $\left(\mu_{\mathrm{c}}+3.3 \cdot \delta_{\mathrm{c}}\right)$ and LOQ $\left(\mu_{\mathrm{c}}+\right.$ $\left.10 \cdot \delta_{\mathrm{c}}\right)$ were 0.014 and $0.032 \mathrm{mV} \mathrm{s}^{-1}$, respectively. LOD 

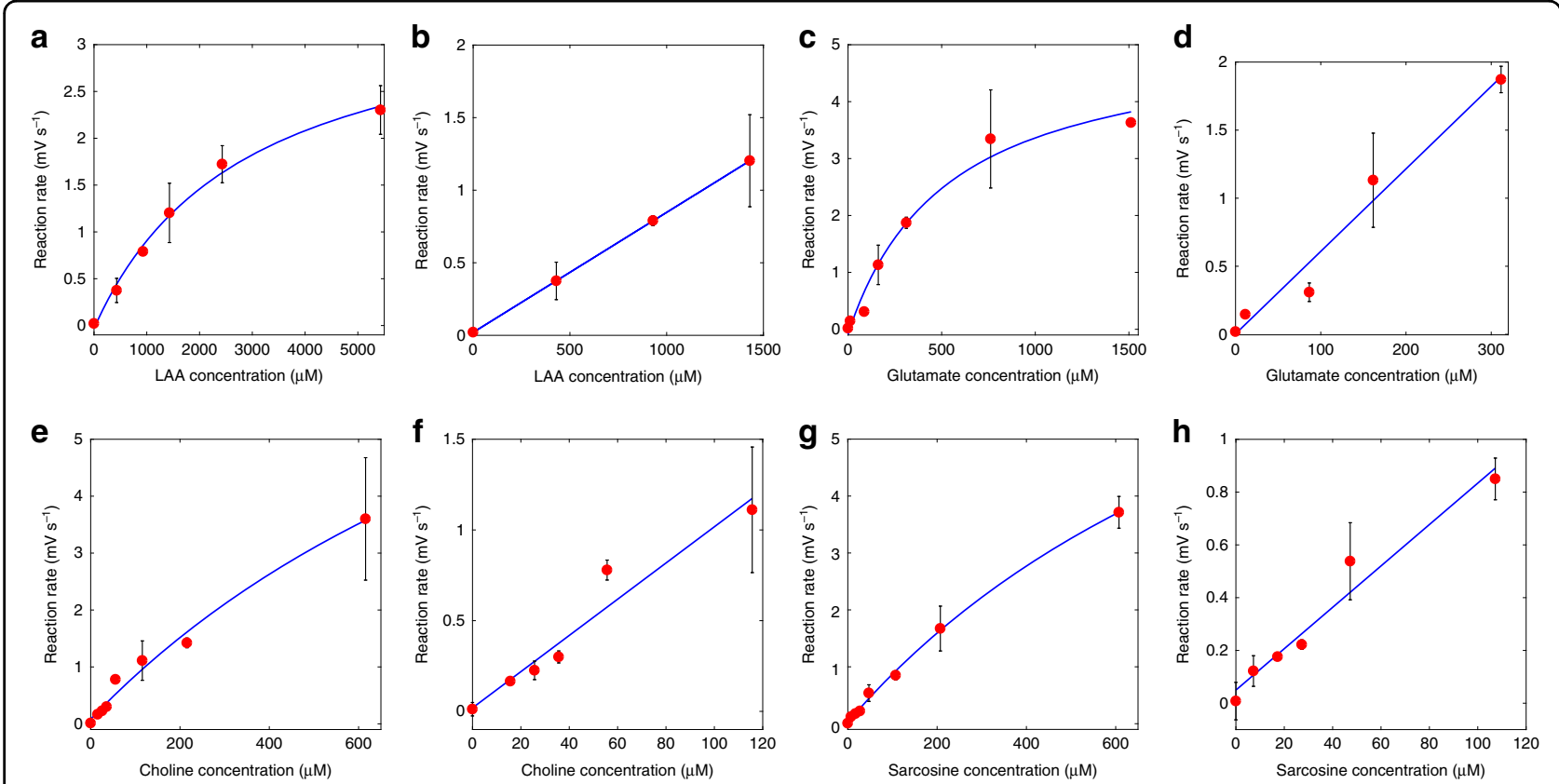

Fig. 3 Calibration curves. Calibration curves obtained by fitting the data with Michaelis-Menten and linear models for $\mathbf{a}, \mathbf{b} L A A, \mathbf{c}$, $\mathbf{d}$ glutamate, e, $\mathbf{f}$ choline, and $\mathbf{g}$, $\mathbf{h}$ sarcosine in human plasma.

and LOQ expressed in $\mathrm{mV} \mathrm{s}^{-1}$ were then converted to $\mu \mathrm{M}$ by using the estimated Michaelis-Menten curve for each metabolite. Thus, the LODs for LAA, glutamate, choline, and sarcosine were $11.1,1.4,1.7$, and $1.4 \mu \mathrm{M}$, respectively. Similarly, the LOQ values for the metabolites in the same order were $25.5,3.3,3.9$, and $3.5 \mu \mathrm{M}$.

\section{Clinical study}

For each sample, the plasma concentrations of LAA, glutamate, choline, and sarcosine were quantified using the experimental platform. The procedures and methods are described in the "Materials and methods" section of this paper.

The average concentration of a metabolite for all samples, including the non-PCa and PCa subjects, is referred to as the grand average. A grand average was calculated for each of the four metabolites we measured. The grand averages were $2421 \pm 952 \mu \mathrm{M}$ for LAA, $53.7 \pm 26.4 \mu \mathrm{M}$ for glutamate, $11.7 \pm 7.0 \mu \mathrm{M}$ for choline, and $10.6 \pm 6.0 \mu \mathrm{M}$ for sarcosine.

The average values measured for each metabolite for the non-PCa and PCa groups were also calculated. For the non-PCa samples, the average concentrations were $1984 \pm 527 \mu \mathrm{M}$ for LAA, $40.2 \pm 11.2 \mu \mathrm{M}$ for glutamate, $10.0 \pm 4.1 \mu \mathrm{M}$ for choline, and $11.5 \pm 4.3 \mu \mathrm{M}$ for sarcosine. The average concentrations of LAA, glutamate, choline and sarcosine in the PCa group were $2694 \pm 1052 \mu \mathrm{M}$, $62.2 \pm 29.5 \mu \mathrm{M}, \quad 13.4 \pm 7.9 \mu \mathrm{M}, \quad$ and $10.0 \pm 6.9 \mu \mathrm{M}$, respectively.
LAA, glutamate, and choline levels were increased in the $\mathrm{PCa}$ group compared with the non-PCa group. There was no relevant cross-correlation between different metabolites, and all cross-correlations were $<0.3$ (Supplementary Information). These data are summarized in Table 2 and presented in Fig. 4.

\section{Multivariate analysis}

To determine the validity of using LAA, glutamate and choline as potential diagnostic markers, the data set was used to train a random forest classification algorithm ${ }^{58}$. The task of the classifier was to provide a binary "negative-or-positive" response to whether a sample was a control (negative) or cancer (positive) sample, using the concentrations of LAA, glutamate and choline as inputs. Using the "randomForest" and "caret" functions in the $\mathrm{R}$ software tool, the algorithm was set to use 500 trees and try up to three metabolites at each split. The model was validated using a repeated "tenfold" procedure that was run 100 times $^{59,60}$. In this way, we generated a crossvalidated receiver operator characteristic (ROC) curve using the predictions over every iteration. For each iteration, a bootstrap resampling procedure was used. The metrics of the classifier were expressed as an average, and a 95\% confidence interval over the distribution was obtained for the 100 independent training and validation iterations. The area under the curve (AUC) was found to be 0.78 , with a $95 \%$ confidence interval of $0.55-0.99$. The ROC curve shows an operating point at a sensitivity of 
Table 1 Platform characterization using human plasma.

\begin{tabular}{|c|c|c|c|c|}
\hline & LAA & Glutamate & Choline & Sarcosine \\
\hline Physiological range & $2-3.5 \mathrm{mM}^{68}$ & $40-150 \mu M^{47}$ & $7-20 \mu \mathrm{M}^{69}$ & $0-20 \mu \mathrm{M}^{50}$ \\
\hline Test range & $0-5.4 \mathrm{mM}$ & $0-1500 \mu \mathrm{M}$ & $0-600 \mu \mathrm{M}$ & $0-600 \mu \mathrm{M}$ \\
\hline Model & \multicolumn{4}{|l|}{$y=\frac{V_{\max } x}{K_{\mathrm{m}}+x}+c$} \\
\hline$V_{\max }\left(\mathrm{mV} \mathrm{s}^{-1}\right)$ & $3.63 \pm 0.51$ & $5.28 \pm 0.93$ & $11.34 \pm 6.9$ & $11.03 \pm 2.1$ \\
\hline$c\left(\mathrm{mV} \mathrm{s}^{-1}\right)$ & $-0.032 \pm 0.126$ & $-0.087 \pm 0.265$ & $0.082 \pm 0.254$ & $0.027 \pm 0.04$ \\
\hline$K_{\mathrm{m}}(\mu \mathrm{M})$ & $2866 \pm 1008.2$ & $529.7 \pm 269.5$ & $1382 \pm 210.7$ & $1209 \pm 335.7$ \\
\hline RMSE & 0.086 & 0.266 & 0.169 & 0.062 \\
\hline$R^{2}$ & 0.994 & 0.979 & 0.985 & 0.998 \\
\hline Linear model & \multicolumn{4}{|l|}{$Y=S \cdot x+C$} \\
\hline Linear range $(\mu \mathrm{M})^{\mathrm{a}}$ & $0-1500$ & $0-320$ & $0-120$ & $0-120$ \\
\hline Sensitivity $(S)\left(\mathrm{mV} \mathrm{s}^{-1} \mathrm{mM}^{-1}\right)$ & $0.83 \pm 0.002$ & $6.06 \pm 1.01$ & $9.98 \pm 1.79$ & $7.84 \pm 1.12$ \\
\hline Baseline $\left(m V s^{-1}\right)$ & $0.020 \pm 15 \cdot 10^{-4}$ & $0.003 \pm 0.163$ & $0.019 \pm 0.1$ & $0.050 \pm 0.056$ \\
\hline RMSE (linear) & $8.6 \cdot 10^{-04}$ & 0.159 & 0.116 & 0.070 \\
\hline$R^{2}$ (linear) & 0.999 & 0.969 & 0.939 & 0.961 \\
\hline Average relative std. dev. (linear) ${ }^{b}$ & $18.3 \%$ & $17.2 \%$ & $16.4 \%$ & $19.2 \%$ \\
\hline Negative control $\left(\mu \vee s^{-1}\right)^{c}$ & \multicolumn{4}{|l|}{$5.0 \pm 2.7$} \\
\hline $\operatorname{LOD}(\mu \mathrm{M})^{\mathrm{d}}$ & 11.1 & 1.4 & 1.7 & 1.4 \\
\hline LOQ $(\mu M)^{d}$ & 25.5 & 3.3 & 3.9 & 3.5 \\
\hline Drift (dark/source on) $\left(\mu \vee s^{-1}\right)$ & \multicolumn{4}{|l|}{$1.4 \pm 1.0 / 0.9 \pm 1.0$} \\
\hline Avg. steady state (dark/source on) (V) & \multicolumn{4}{|c|}{$0.486 \pm 0.003 / 1.730 \pm 0.031$} \\
\hline
\end{tabular}

a Linear range is here defined as the measurement range where the linear model had $R^{2}>0.9$.

${ }^{b}$ Average of the standard deviation of the measurements in the linear range.

cAverage over 24 measurements.

${ }^{\mathrm{d}}$ Converted from $\mathrm{mV} \mathrm{s}^{-1}$ to $\mu \mathrm{M}$ using the Michaelis-Menten model.

0.94 , with a $95 \%$ confidence interval of $0.82-1.00$, and a specificity of 0.70 , with a $95 \%$ confidence interval of 0.40-0.98, as shown in Fig. 5. The diagnostic capability of the classifier can be compared with that of PSA. In clinical practice, the PSA sensitivity and specificity are 0.32 and 0.87 , respectively, for a PSA threshold of $3.1 \mathrm{ng} \mathrm{mL}^{-161}$. PSA yields an AUC of $0.68^{61,62}$. Our results show that the random forest model based on LAA, glutamate, and choline could substantially reduce the number of falsepositive results.

These results show that the platform has the potential to deliver higher diagnostic capability than PSA. We also note that if both the PSA and metabolomic test were to be used together, it would be independently possible to secure both a high sensitivity of up to $94 \%$ (metabolites) and high specificity of $87 \%$ (PSA) to yield a powerful and highly discriminating diagnostic method.

\section{Simultaneous measurements}

To make a practically useful POC diagnostic tool using the markers LAA, glutamate and choline, it is desirable to perform simultaneous multimetabolite measurements. Simultaneous measurements require reagents to be preloaded into the microfluidic channels. Sarcosine was excluded from these experiments since it was concluded that it was not a useful biomarker for this population.

The performance of the platform with preloaded dried reagents (see Materials and Methods section) was assessed by obtaining calibration curves for plasma LAA, glutamate, and choline in the microfluidic device. Each metabolite was tested individually in undiluted human plasma spiked with the metabolite to the desired test concentration. The resulting calibration curves are shown in Fig. $6 \mathrm{a}-\mathrm{c}$. The results were similar to the calibration curves obtained from using off-chip mixing of the liquid reagents. A comparison between the two test methods is shown in Table 3. The linearity in the physiological range was nearly the same, but a slight loss of sensitivity for LAA and choline when using the dried reagents was observed. The control channel, which contained dried dye and peroxidase only, showed a measurable response when compared to photodiodes with no dried assay material 
Table 2 Clinical study results in the control and cancer groups.

\begin{tabular}{|c|c|c|c|c|}
\hline & LAA & Glutamate & Choline & Sarcosine \\
\hline \multicolumn{5}{|l|}{ Overall data set } \\
\hline Grand average \pm std. dev. $(\mu \mathrm{M})$ & $2421 \pm 952$ & $53.7 \pm 26.4$ & $11.7 \pm 7.0$ & $10.6 \pm 6.0$ \\
\hline Grand median $(\mu \mathrm{M})$ & 2072 & 47.9 & 10.0 & 9.9 \\
\hline Range $(\mu \mathrm{M})$ & $1213-5421$ & $6.3-149.5$ & $2.3-36.9$ & $1.7-27.2$ \\
\hline Temperature $\left({ }^{\circ} \mathrm{C}\right)$ & $27.3 \pm 1.0$ & $26.4 \pm 1.3$ & $26.3 \pm 0.9$ & $25.9 \pm 1.2$ \\
\hline Humidity (\%) & $52.6 \pm 5.0$ & $49.5 \pm 7.8$ & $44.4 \pm 9.0$ & $42.2 \pm 10.5$ \\
\hline \multicolumn{5}{|l|}{ Non-PCa group } \\
\hline Non-PCa average \pm std. dev. $(\mu \mathrm{M})$ & $1984 \pm 527$ & $40.2 \pm 11.2$ & $10.0 \pm 4.1$ & $11.5 \pm 4.3$ \\
\hline Non-PCa median ( $\mu \mathrm{M})$ & 1966 & 39.8 & 9.0 & 12.3 \\
\hline Range $(\mu \mathrm{M})$ & $1213-3167$ & $21.9-67.1$ & $2.3-15.4$ & $5.1-18.8$ \\
\hline \multicolumn{5}{|l|}{ PCa group } \\
\hline PCa average \pm std. dev. $(\mu M)$ & $2694 \pm 1052$ & $62.2 \pm 29.5$ & $13.4 \pm 7.9$ & $10.0 \pm 6.9$ \\
\hline PCa median $(\mu \mathrm{M})$ & 2386 & 61.0 & 10.4 & 9.7 \\
\hline Range $(\mu \mathrm{M})$ & $1503-5410$ & $6.3-149.5$ & $4.7-36.9$ & $1.7-27.2$ \\
\hline \multicolumn{5}{|l|}{ Univariate analysis } \\
\hline PCa/non-PCa (average) & 1.36 & 1.55 & 1.34 & 0.87 \\
\hline PCa/non-PCa (median) & 1.21 & 1.53 & 1.15 & 0.79 \\
\hline$t$-test ( $p$-value) & 0.03 & 0.02 & 0.06 & 0.27 \\
\hline
\end{tabular}

present. The observed control signal was still small compared to the signals observed in the test channels and gave rise to the increase in the calculated LOD and LOQ for all the target metabolites. Under these conditions, the LODs for plasma LAA, glutamate, and choline were 42.9, 6.4 , and $3.2 \mu \mathrm{M}$, respectively. Similarly, the LOQ values for the metabolites, in the same order, were 129.3, 19.5, and $9.8 \mu \mathrm{M}$.

To evaluate channel-to-channel independence on the same chip, the four channels in a set of 12 cartridges were filled with dried reagents for LAA, glutamate, choline and a negative control. Using one cartridge at a time, triplicates of each of the following were measured by flowing the sample into the channels: DI water; $250 \mu \mathrm{M}$ choline in DI water; $250 \mu \mathrm{M}$ glutamate in DI water; and $2.5 \mathrm{mM}$ LAA in DI water. There was no response to DI water only, and as expected, each functionalized channel only responded to the metabolite for which it had been prepared.

A further triplicate of cartridges was prepared with the three functionalized channels and a control, and in each, an unmodified human plasma sample was introduced to the cartridge. The plasma sample yielded signal rates above the LOQ for LAA, glutamate, and choline measurements. No obvious crosstalk was observed in these experiments, and the results are summarized in Fig. 6d.
A proof of principle clinical validation of the platform for multimetabolite testing using dried reagents in a single cartridge was then conducted using one individual each from the non-PCa and PCa sample groups. Simultaneous readings for different metabolites are shown in Fig. 7. For both clinical samples, the reaction rates were found to be similar to the respective wet assay. The rates obtained with dried and liquid reagents were well correlated with $R^{2}>0.91$.

The rates obtained with dried reagents for non-PCa and PCa samples were also compared; the rates were consistently higher for the PCa sample, as was the case with the wet assays. This provides a proof of principle that the platform can provide clinically relevant information when testing for the metabolites of interest simultaneously.

\section{Discussion}

We have shown that the method for acquiring data using multiple metabolites can be integrated into a silicon chip-based device capable of making all the measurements simultaneously. Using the device, a preliminary clinical study demonstrated that a model can be created using multiple metabolites to discriminate patients with PCa from normal controls. LAA, glutamate and choline showed a significant correlation in our population, and the data were used to train a random forest classification 

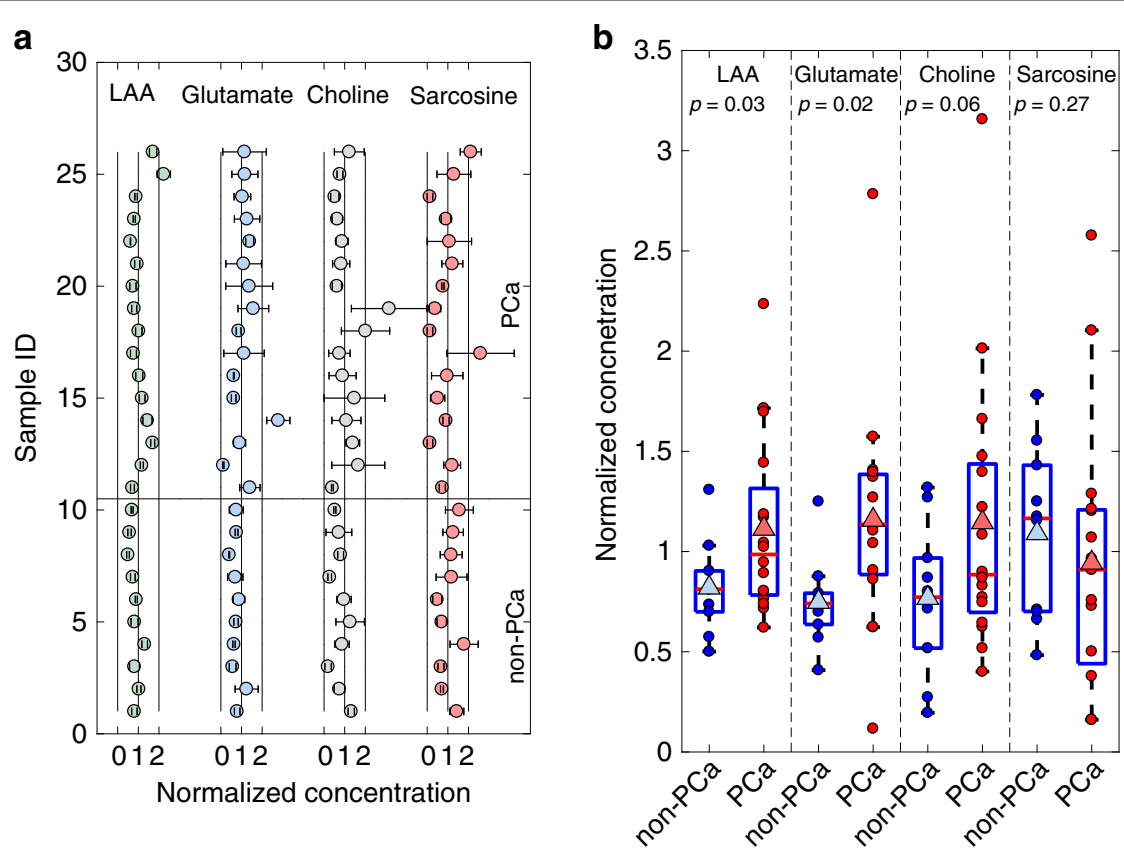

Fig. 4 Results of the clinical study. a Metabolite quantification in the non-PCa (samples 1-10) and PCa groups (samples 11-26). The concentration of the metabolic biomarkers was normalized to the grand average. $\mathbf{b}$ Univariate analysis and box plots for the non-PCa group vs the PCa group. Blue markers indicate non-PCa measurements. Red markers indicate PCa measurements. The triangular markers indicate the average values in the nonPCa and PCa groups.

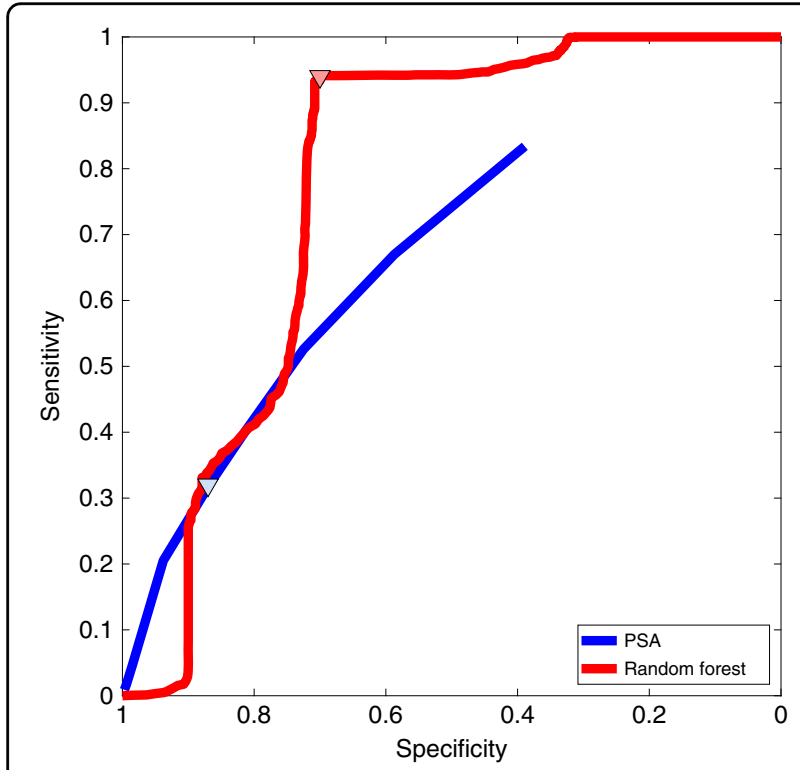

Fig. 5 Multivariate analysis. ROC curve for the cross-validated random forest algorithm trained with the measured metabolic profiles of LAA, glutamate, and choline. The ROC curve is compared with that for $P S A^{61}$. Operating points are shown using triangular markers.

model. In our study, sarcosine did not show any relevant correlation with $\mathrm{PCa}$; therefore, the data were not used for the classifier. The ROC curve for the new test was found to have an $\mathrm{AUC}=0.78$ that compared favorably with an AUC $=0.68$ for the PSA test. The test ROC curve intersects the PSA ROC curve near the standard threshold with a specificity of $\sim 87 \%$ and a sensitivity of $32 \%$. However, the new test's ROC curve has a significantly greater sensitivity of $94 \%$ when operated at a slightly lower specificity of $70 \%$.

The metabolic biomarker panel we present provides a valuable proof-of-concept and can potentially be improved by including additional metabolites. Table 3 in the Supplementary Information presents additional $\mathrm{PCa}$ related blood metabolites that could be quantified using the platform. The availability of data on a larger marker panel will enable a comprehensive analysis of the proposed diagnostic method. Further work with a larger population of subjects leading to a full clinical trial using the diagnostic method proposed in this paper will be necessary to demonstrate that the platform can deliver an effective POC diagnostic tool for PCa.

We envisage a potential scale-up to build a system capable of measuring a whole-person metabolome in a single measurement from a drop of blood. The procedures and methods developed in this work can be optimized to improve the LOD and LOQ and applied to a larger CMOS sensor array to deliver increased multiplexing capabilities. Future work will include developing methods for highly dense measurement multiplexing with low crosstalk. The technology may also take advantage of 

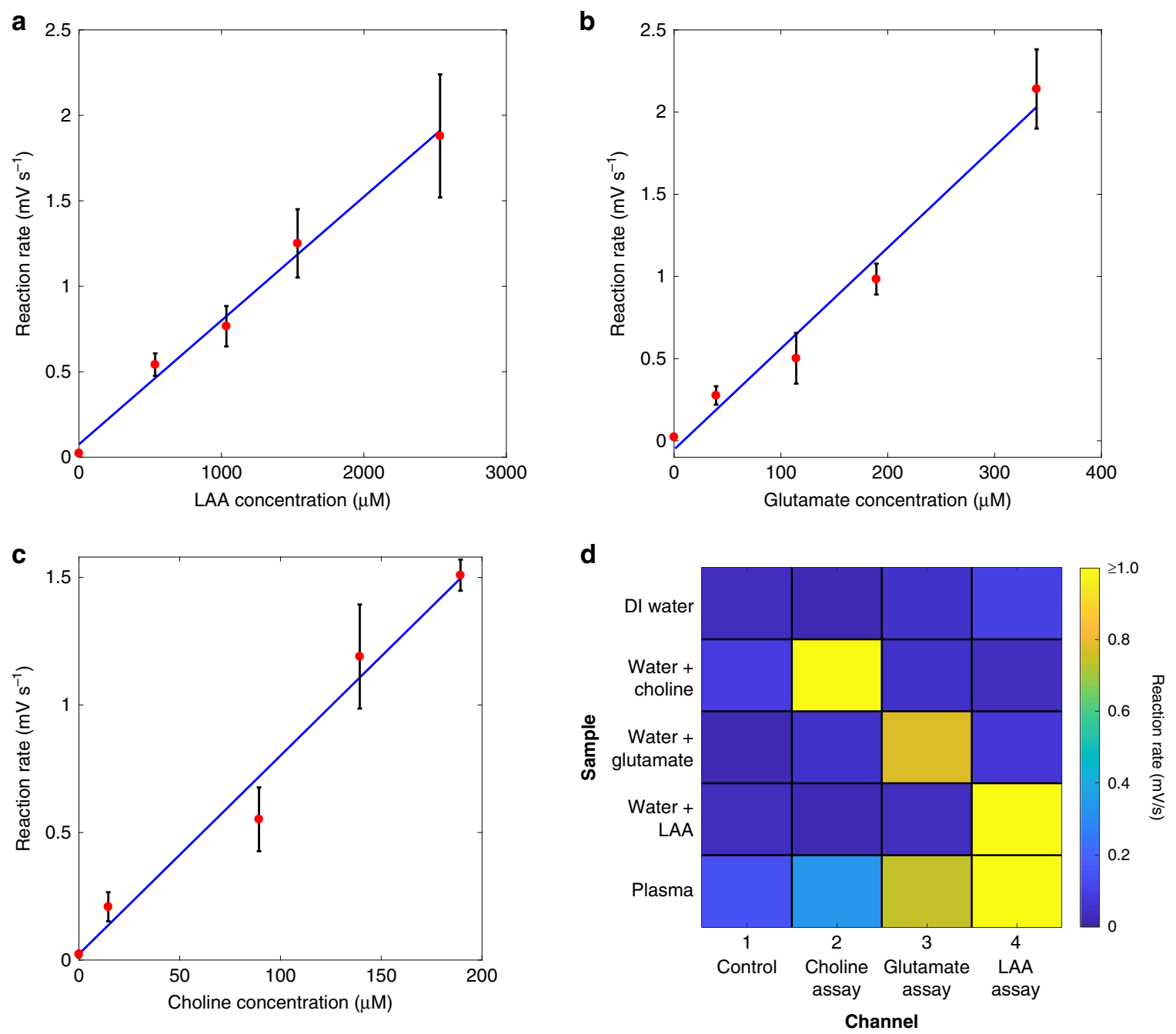

Fig. 6 Assays with preloaded reagents. Calibration curves for plasma $\mathbf{a} L A A$, $\mathbf{b}$ glutamate, and $\mathbf{c}$ choline obtained with reagents preloaded in the microfluidic channels in the dried state. $\mathbf{d}$ Control experiments to evaluate the potential crosstalk for multimetabolite sensing. Metabolites in plasma have different physiological concentrations. Hence, there is a difference in the absolute value of the reaction rate. The blue sectors in the first four lines indicate that there was very little crosstalk.

the computation capabilities of CMOS to create a device capable of not only collecting raw data but also integrating complete machine learning algorithms to yield a highly sensitive general-purpose chip, or chip-family, capable of extracting large amounts of highly specific and individualized data.

\section{Conclusion}

Survival rates for many types of cancer are continuing to improve $^{63}$, but progress towards improving the outcome for men with prostate cancer has been hindered by the need for a reliable test. The lack of such a test has inhibited the introduction of mass screening programs. As a consequence, many instances of cancer are only detected very late, when the possibilities for effective treatment are reduced. The CMOS point-of-care platform presented in this paper has the potential to address this problem by improving the accuracy of a diagnostic test to such an extent that screening will become a more clear-cut choice. Future tests may combine the merits of more than one assay; hence, metabolite measurements could be used in conjunction with a test for PSA. Indeed, progress is also being made to develop POC tests for PSA ${ }^{16}$. We provide proof-of-concept for a POC platform using a CMOS sensor chip with monolithically integrated microfluidics that is capable of performing multiple metabolite tests pertinent to the diagnosis of prostate cancer simultaneously. The system was shown to be capable of detecting diagnostically significant information in the population under test and can be used to improve the current clinical 
Table 3 Comparison of the performance of the platform for the quantification of LAA, glutamate, and choline when using off-chip mixed liquid reagents or preloaded dried reagents.

\begin{tabular}{|c|c|c|c|c|c|c|}
\hline \multirow{3}{*}{$\begin{array}{l}\text { Physiological range: } \\
\text { Reagents: }\end{array}$} & \multirow{2}{*}{$\begin{array}{l}\text { LAA } \\
2-3.5 \mathrm{mM}^{68}\end{array}$} & \multicolumn{3}{|c|}{ Glutamate } & \multicolumn{2}{|c|}{ Choline } \\
\hline & & & $40-150 \mu \mathrm{M}^{4}$ & & $7-20 \mu l$ & \\
\hline & Liquid & Dried & Liquid & Dried & Liquid & Dried \\
\hline Sensitivity $\left(\mathrm{mV} \mathrm{s}^{-1} \mathrm{mM}^{-1}\right)$ & $0.83 \pm 0.002$ & $0.72 \pm 0.07$ & $6.06 \pm 1.01$ & $6.14 \pm 0.87$ & $9.98 \pm 1.79$ & $7.78 \pm 1.13$ \\
\hline Linearity $\left(R^{2}\right)$ & 0.999 & 0.991 & 0.969 & 0.977 & 0.939 & 0.975 \\
\hline RMSE & 0.086 & 0.076 & 0.266 & 0.145 & 0.169 & 0.115 \\
\hline Average std. dev. (\%) & $18.3 \%$ & $15.7 \%$ & $17.2 \%$ & $18.0 \%$ & $16.4 \%$ & $17.7 \%$ \\
\hline Negative control $\left(\mu \vee s^{-1}\right)$ & $5.0 \pm 2.7$ & $23.0 \pm 12.5$ & $5.0 \pm 2.7$ & $23.0 \pm 12.5$ & $5.0 \pm 2.7$ & $23.0 \pm 12.5$ \\
\hline LOD $(\mu \mathrm{M})$ & 11.1 & 42.9 & 1.4 & 6.4 & 1.7 & 3.2 \\
\hline LOQ $(\mu \mathrm{M})$ & 25.5 & 129.3 & 3.3 & 19.5 & 3.9 & 9.8 \\
\hline
\end{tabular}

standard. Furthermore, the platform has the potential to be used in a domestic environment and is therefore capable of detecting early changes in candidate biomarkers when measured over a period of time. The technology presented in this article has wide-reaching implications, not only for cancer, as illustrated in Table 1 of the Supplementary Information but for other diseases and personalized medicine. Metabolite marker panels are now described for illnesses including sepsis ${ }^{64}$, acute kidney injury $^{65}$, and cardiovascular disease ${ }^{66}$. We anticipate a future microelectronic platform to exploit the scalable properties of CMOS that will become as commonplace in medicine as the stethoscope and thermometer are today.

\section{Materials and methods Microfluidic design}

Microfluidic channels were designed to provide laminar and passive flow. The geometry of the design, composed of straight microchannels with rectangular cross-sections, was chosen to match the layout of the CMOS sensor array. Custom MATLAB simulations were carried out to identify the dimensions of the microchannels needed to yield higher capillary pressure and lower filling time in the laminar flow regime.

\section{Microfluidics integration}

Microfluidics was integrated with the CMOS chip by replica and injection molding in five stages: (1) SU-8 mold fabrication, (2) PDMS mold fabrication, (3) wire bonding, (4) epoxy encapsulation, and (5) channel enclosure.

1. A silicon wafer $\left(4^{\prime \prime}\right)$ was cleaned with IPA, acetone, and DI water; sonicated; dehydrated $\left(10 \mathrm{~min}, 90^{\circ} \mathrm{C}\right)$; and plasma-oxidized ( $2 \mathrm{~min}, 120 \mathrm{~W}$ ). A first SU-8 3050 layer was spin-coated onto the wafer $(30 \mathrm{~s}$, $1000 \mathrm{rpm}$ ) and baked (90 $\mathrm{min}, 90^{\circ} \mathrm{C}$ ). A second SU-8
3050 layer was similarly spin-coated and baked. The substrate was exposed twice to UV light using a mask aligner (70 s each time, $15 \mathrm{~s}$ wait time). Afterward, the sample was baked $\left(10 \mathrm{~min}, 90^{\circ} \mathrm{C}\right)$, developed using EC solvent (for $28 \mathrm{~min}$ ), rinsed with IPA, and baked $\left(30 \mathrm{~min}, 180^{\circ} \mathrm{C}\right.$ ).

2. The SU-8 mold was silanized by exposure to trichlorosilane (30 min in an evacuated chamber) and placed into a petri dish. PDMS (25 g, 1:14 ratio) was poured onto the mold, degassed $(1 \mathrm{~h}$ in a vacuum chamber), and cured $\left(2 \mathrm{~h}, 70^{\circ} \mathrm{C}\right)$. Cured PDMS was released from the SU-8 mold, placed on a clean substrate, cut with a sharp knife, aligned, and temporarily bonded to the CMOS chip using a flipchip bonder (model 850, Semiconductor Equipment Corp.). The bond strength was increased by heating the two respective part holders for the chip and PDMS $\left(90^{\circ} \mathrm{C}, 10 \mathrm{~min}\right)$ under constant pressure (5 psi).

3. The CMOS chip with the bonded PDMS mold was glued into the $8.3 \times 8.3 \mathrm{~mm}$ cavity at the center of a 120 pin ceramic chip pin grid array package using EPO-TEK H74 epoxy (Epoxy Technology Inc.) and wire-bonded (by Hesse and Knipps Bondjet 710).

4. A black epoxy resin (302-3M 1LB, Epoxy Technology Inc.) was injected into the PDMS microstructure and cured $(48 \mathrm{~h}$ at room temperature). After curing, the PDMS structure was removed from the chip. Because there were no wire bonds on the top and bottom edges of the chip, microchannels were extended in these directions, effectively planarizing the surface. Epoxy also provided encapsulation of the wire bonds.

5. A planar slab of PDMS was cut with a sharp knife $(4 \times 3 \mathrm{~mm})$, cleaned, exposed to oxygen plasma 

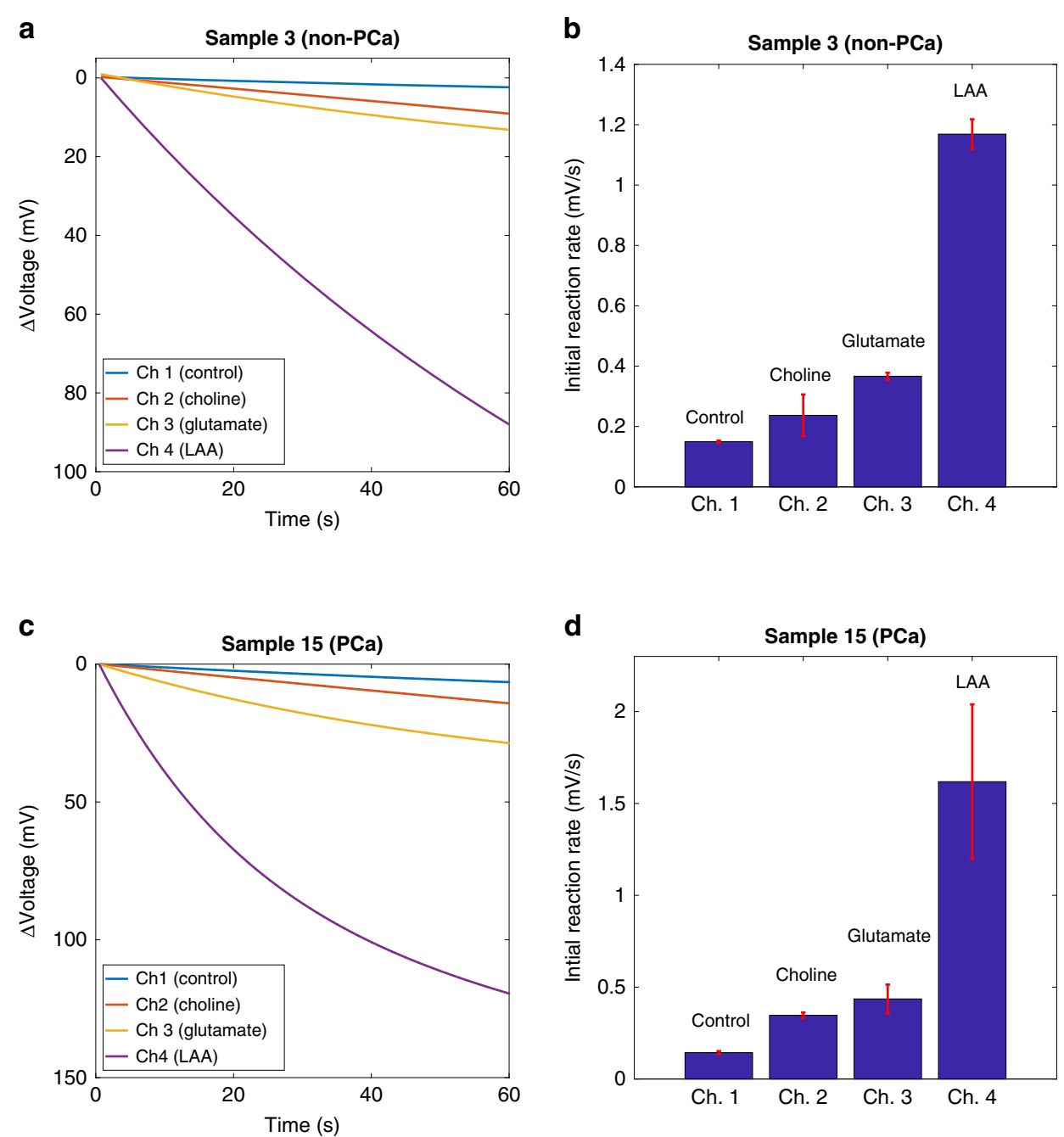

Fig. 7 Simultaneous testing with clinical samples. a Initial reaction rates in four different microfluidic channels for a non-PCa sample. b Output from different microchannels for a non-PCa sample. $\mathbf{c}$ Initial reaction rates in four different microfluidic channels for a PCa sample. $\mathbf{d}$ Output from different microchannels for a PCa sample.

$(1 \mathrm{~min}, 80 \mathrm{~W})$ and immersed in a PVA solution $(1 \mathrm{wt} \%)^{55}$. The PVA-modified PDMS slab was permanently bonded to the epoxy microstructure by plasma activation $(45 \mathrm{~s}, 80 \mathrm{~W})$ and baking $\left(15 \mathrm{~min}, 90^{\circ} \mathrm{C}\right)$.

The approach that was used encapsulated all the watersensitive electronic components on the cartridge in epoxy. This enabled leakage-free aqueous experiments on the cartridge. A graphical representation of the fabrication is shown in Fig. 3 of the Supplementary Information.

\section{Reagents}

All chemicals required for the assays were purchased from Sigma-Aldrich unless otherwise specified. A reagent solution per target metabolite was prepared immediately before the experiment. Assay formulations were optimized by experimentation. All the reagents were prepared in DI water.
For LAA testing, $6.7 \mu \mathrm{L}$ of LAAOX $\left(10 \mathrm{U} \mathrm{mL}^{-1}\right)$, $6.7 \mu \mathrm{L}$ of $\mathrm{HRP} \quad\left(150 \mathrm{U} \mathrm{mL}^{-1}\right), \quad 3.3 \mu \mathrm{L}$ of phenol $(44.5 \mathrm{mM})$ and $3.3 \mu \mathrm{L}$ of 4 -aminoantipyrine (4-AAP, $10.5 \mathrm{mM}$ ) were mixed. The reagent solution for glutamate was prepared by mixing $6.7 \mu \mathrm{L}$ of GLOX $(4 \mathrm{U}$ $\left.\mathrm{mL}^{-1}\right), 6.7 \mu \mathrm{L}$ of HRP $\left(150 \mathrm{U} \mathrm{mL}^{-1}\right), 3.3 \mu \mathrm{L}$ of phenol (44.5 mM), and $3.3 \mu \mathrm{L}$ of 4 -AAP $(10.5 \mathrm{mM})$. For choline testing, $6.7 \mu \mathrm{L}$ of $\mathrm{CHOX}\left(150 \mathrm{U} \mathrm{mL}^{-1}\right), 6.7 \mu \mathrm{L}$ of HRP $\left(300 \mathrm{U} \mathrm{mL}^{-1}\right), 3.3 \mu \mathrm{L}$ of phenol $(44.5 \mathrm{mM})$, and $3.3 \mu \mathrm{L}$ of 4-AAP $(10.5 \mathrm{mM})$ were mixed. The reagent solution for sarcosine was prepared by mixing $6.7 \mu \mathrm{L}$ of SAOX $\left(200 \mathrm{U} \mathrm{mL}^{-1}\right), 6.7 \mu \mathrm{L}$ of HRP $\left(300 \mathrm{U} \mathrm{mL}^{-1}\right), 3.3 \mu \mathrm{L}$ of phenol $(44.5 \mathrm{mM})$, and $3.3 \mu \mathrm{L}$ of 4 -AAP $(10.5 \mathrm{mM})$. The reagent solution for the negative control was prepared by mixing $6.7 \mu \mathrm{L}$ of DI water, $6.7 \mu \mathrm{L}$ of HRP $\left(300 \mathrm{U} \mathrm{mL}^{-1}\right), 3.3 \mu \mathrm{L}$ of phenol $(44.5 \mathrm{mM})$, and $3.3 \mu \mathrm{L}$ of 4-AAP $(10.5 \mathrm{mM})$. 


\section{Experimental setup}

To ensure consistent results, a rigid test setup was built. All optomechanical components were purchased from Thorlabs. The reader of the platform was secured to an optical aluminum breadboard $(15 \mathrm{~cm} \times 10 \mathrm{~cm} \times 1.2 \mathrm{~cm})$ with the ZIF socket facing up. Cartridges were inserted into the ZIF socket. The sensitive area of the CMOS chip was parallel to the optical breadboard and facing up. Using optomechanics, a $3 \mathrm{~mW}$ LED $(\lambda=490 \mathrm{~nm}$, FWHM $=20 \mathrm{~nm}$ ) powered using a power supply unit (HP E3631A) was used to uniformly illuminate the sensing area on the CMOS chip with a collimating lens (AC254035-A-ML BBAR coating $f=35 \mathrm{~mm}$ ). The height and positioning of the LED and lens were adjusted so that the sensitive area of the chip received perpendicular collimated light. The equipment that was mounted onto the optical breadboard was enclosed in a box and covered with a nylon/polyurethane blackout cloth. The cloth was essential to ensure that the experiments were performed in a dark environment. A small opening was left for connecting wires and allowed sample delivery to the chip. The reader, enclosed in the dark environment, was connected to an external laptop (HP EliteBook i7-8650u 16 GB) using a USB cable. The MATLAB-based user interface, running on the laptop, was used to control data acquisition. Data were recorded with an average frame rate of $36.5 \mathrm{fps}$ and a resolution of 12 bits. Typically, the duration of a single experiment was $5 \mathrm{~min}(\sim 10,950$ frames). Environmental temperature and humidity were also monitored during testing using a Texas Instrument module (HDC 1080EVM).

\section{Cartridge reusage}

Although the cartridge could in principle be a single-use disposable device, because of limited resources, in this work, cartridges were cleaned and reused. A cleaning procedure after every measurement was used to avoid cross-contamination. The cleaning process involved a sequential rinse in DI water, IPA, and then ethanol, and nitrogen was used to blow it dry. For the clinical samples, an additional first rinse step with a dilute piranha solution was performed. The dilute piranha solution was prepared using 10:3:1 DI water:18 M sulfuric acid:30\% hydrogen peroxide. Cleaning the cartridge with dilute piranha was kept to a minimum since the etchant attacked the epoxy microchannels. Cross-contamination reduction was also achieved by optimizing the testing sequence. In particular, one or more negative controls were performed before any measurements.

\section{Calibration}

For calibration with liquid reagents, one human plasma sample was purchased from Sigma-Aldrich and reconstituted according to the manufacturer's instructions.
Subsequently, it was modified by adding known quantities of analytes of interest. Additional concentrations did not take into account the unknown endogenous level of the substrate of interest in the sample. The endogenous concentration was estimated by using the method for substrate measurement described in the signal processing section. Twenty microliters of reagent solution was mixed off-chip with $20 \mu \mathrm{L}$ of sample and introduced into the cartridge within a few seconds.

For calibration with dried reagents, human plasma was purchased, reconstituted, and modified using the same procedure described for calibration with liquid reagents. The same reagents were preloaded in all four microchannels in a single cartridge. Thirty microliters of sample was introduced to the cartridge without any further dilution.

For both configurations, metabolites were tested individually. Each cartridge had four microchannels; therefore, each measurement yielded four reaction rates. The method employed for sample delivery was reliable and repeatable; therefore, air bubbles or fluidic failures did not pose a problem for the majority of the experiments. However, in a small number of instances, unexpected behavior was observed, and the data were excluded. The four reaction rates were averaged ${ }^{67}$, and the small number of anomalies that occurred was mitigated using triplicate measurements from each cartridge. Each cartridge was functionalized with dried reagents prior to each new measurement. The errors were expressed using the standard deviation of these data.

\section{Non-PCa samples}

Ten samples of human plasma from healthy people, herein referred to as "non-PCa", were commercially sourced from Cambridge Bioscience. The exclusively adult male non-PCa donors were age $34 \pm 10$ years. The ethnicity of the group was diversified. The samples were tested for the most common infectious diseases, and all gave negative results. Approximately $10 \mathrm{~mL}$ of fresh blood samples were collected from subjects in various research centers in England, mixed with $10 \mathrm{mg}$ of $\mathrm{K}_{2}$ EDTA anticoagulant and centrifuged. The resulting 4-mL plasma samples were frozen at $-80^{\circ} \mathrm{C}$ and shipped under dry ice. After collection, plasma samples were aliquoted and stored at $-80^{\circ} \mathrm{C}$. No additional freeze and thaw cycle was performed.

\section{PCa samples}

Sixteen samples of human plasma from people diagnosed with PCa, herein referred to as the "PCa group", were sourced from the Beatson West of Scotland Cancer Centre, Glasgow, UK, using an ethically approved sample collection protocol. Donors were adults already diagnosed with PCa. All patients were under treatment. 
Approximately $10 \mathrm{~mL}$ of blood samples was collected at the cancer center, mixed with $10 \mathrm{mg}$ of $\mathrm{K}_{2}$ EDTA anticoagulant, and centrifuged, and the resulting plasma samples were frozen at $-80^{\circ} \mathrm{C}$. Samples were transported in dry ice. Afterward, plasma samples were aliquoted and stored at $-80^{\circ} \mathrm{C}$. No additional freeze and thaw cycle was performed. A copy of the ethical approval letter and consent forms from the donors are available upon request.

\section{Preliminary clinical study}

The non-PCa and PCa groups were tested for the four metabolites of interest for PCa diagnosis. Chronologically, the non-PCa group was tested before the $\mathrm{PCa}$ group. For convenience numbered sample IDs were assigned. Samples with IDs from 1 to 10 belong to the non-PCa group. Samples with sample IDs from 11 to 26 belong to the PCa group.

Within each group, metabolites were measured oneby-one in the following order: LAA, glutamate, choline, and sarcosine. Experiments were performed with wet reagents. Twenty microliters of reagent solution was offchip mixed with $20 \mu \mathrm{L}$ of clinical sample and introduced onto the platform within a few seconds. For each sample and metabolite, the negative control (background) was first assessed. Then, the assay was performed. Finally, positive controls $\mathrm{A}$ and $\mathrm{B}$ were tested. Additional concentrations of positive control A for LAA, glutamate, choline, and sarcosine were $[\mathrm{A}]=500 \mu \mathrm{M}, 100 \mu \mathrm{M}$, $100 \mu \mathrm{M}$, and $100 \mu \mathrm{M}$, respectively. The additional concentration for positive control $\mathrm{B}$ was $[\mathrm{B}]=2[\mathrm{~A}]$. Controls were obtained using single measurements. The assay was repeated three times using the same cartridge. Averages and standard deviations were obtained for the three measurements.

\section{Microchannel functionalization with dried reagents}

To functionalize the microchannels with dried assay material, reagent solutions for the control and for LAA, glutamate, and choline assays were first prepared as described above. One microliter of each reagent solution was preloaded into the required microchannel by manual pipetting. Ultralong microloader pipette tips (Eppendorf) with an outer diameter of $100 \mu \mathrm{m}$ were used under a microscope. The pipette tips made it possible to dispense the reagent directly into single microchannels. To avoid contamination of the shared input fluidic region, reagent solutions were inserted from the fluidic output-end of the channels. After the deposition of the reagent solutions, the cartridge was dried for $1 \mathrm{~h}$ at room temperature in a vacuum chamber. The control channel was preloaded with a reagent solution containing HRP, phenol, and 4AAP. The presence of dried reagents on the chip slightly increased the light absorbance of the platform after settling. To compensate, the intensity of the light from the LED was increased to keep the PDs at the same operating point with respect to the unfunctionalized microchannels.

Reagents were rehydrated when the sample was introduced into the microchannels. Based on visual inspection of the data from the single sensors, we found that the reagents were distributed uniformly along the sensor region of the channel and remained so after drying.

\section{Simultaneous measurements with clinical samples}

A cartridge with four microchannels was used. One microchannel was functionalized as a negative control. The remaining three channels were functionalized for LAA, glutamate, and choline assays. Fifteen microliters of clinically sourced human plasma samples were introduced into the cartridge with the preloaded reagents without any further dilution. Experiments were repeated twice. Microchannels were functionalized with dried reagents prior to each experiment. Experiments were performed immediately after completing cartridge functionalization.

\section{Signal processing}

Signal processing can be divided into initial reaction rate determination followed by substrate concentration estimation.

To determine the initial reaction rate in a single microchannel, data were first visually inspected. Signals from sensing elements inside the same microfluidic channel were low pass filtered (normalized cutoff frequency: $0.1 ; 8$ th order) and spatially averaged (48 different sensors). Unresponsive sensors or sensors affected by strong artifacts were excluded from the averaging process. The resulting signal was then temporally averaged in 1-s nonoverlapping windows and fitted using a double exponential derived by the Michaelis-Menten model and Beer-Lambert law. The initial rate of the reaction was then calculated by differentiation of the measured signal.

The substrate concentration estimation was performed using the initial reaction rate and sample-specific parameters. For each sample, a negative control reaction was initiated between the sample, peroxidase, and colorchanging reagents with no substrate-specific enzyme present to quantify nonspecific activity. The reaction rate obtained from the negative control $\left(r_{\mathrm{n}}\right)$ was used as a background to adjust the reaction rate of the actual test $\left(r_{\mathrm{t}}\right)$, as follows:

$$
r_{\mathrm{t}}^{*}=r_{\mathrm{t}}-r_{\mathrm{n}}
$$

where $r_{\mathrm{t}}^{*}$ is the adjusted reaction rate of the test. Subtraction of the background can affect the performance of the assay, including the dynamic range, LOD and LOQ. However, each plasma sample used in this work had a 
different background since they came from different individuals. Cartridge to cartridge variations were also expected. The background correction takes into account these variations to yield comparable results.

The sensitivity was estimated using the two positive controls $\mathrm{A}$ and $\mathrm{B}$, where known substrate concentrations $[A]$ and $[B]$, respectively, were added to the undiluted sample. The controls with concentrations $[\mathrm{A}]$ and $[\mathrm{B}]$ gave respective initial reaction rates $r_{\mathrm{a}}$ and $r_{\mathrm{b}}$ in the linear operating range of the platform. The rates $r_{\mathrm{a}}$ and $r_{\mathrm{b}}$ provided the sample-specific sensitivity $\left(S^{\prime}\right)$ of the apparatus according to the following formula:

$$
S^{\prime}=\frac{r_{\mathrm{b}}-r_{\mathrm{a}}}{[\mathrm{B}]-[\mathrm{A}]} \text { where }[\mathrm{B}]>[\mathrm{A}] \text { and } r_{\mathrm{b}}>r_{\mathrm{a}}
$$

By analogy, the sensitivity was also calculated using the following variants:

$$
S^{\prime \prime}=\frac{r_{\mathrm{b}}-r_{\mathrm{t}}}{[\mathrm{B}]-[\mathrm{T}]} ; S^{\prime \prime \prime}=\frac{r_{\mathrm{a}}-r_{\mathrm{t}}}{[\mathrm{B}]-[\mathrm{T}]}
$$

where $T$ is the test sample with an unknown metabolite concentration [T]. Typically, we found that $S^{\prime}, S^{\prime \prime}$, and $S^{\prime \prime \prime}$ had similar numerical values. Their average $(S)$ was then used for substrate quantification. Note that it was not necessary to adjust $r_{\mathrm{a}}$ and $r_{\mathrm{b}}$ using $r_{\mathrm{n}}$ since $r_{\mathrm{n}}$ automatically cancels when computing the difference. $[\mathrm{T}]$ was estimated using linear regression to be:

$$
[\mathrm{T}]=\frac{r_{\mathrm{t}}^{*}}{S}
$$

Additional details regarding signal processing can be found in the Supplementary Information.

\section{Acknowledgements}

This work was supported by EPSRC grants EP/K021966/1 and EP/L023652/1. S.B.P. is now at the Department of Electronic Engineering \& York Biomedical Research Institute, University of York, York, YO10 5DD UK. M.P.B. is part of the Wellcome Centre for Integrative Parasitology funded by a Wellcome Trust core grant (104111/Z/14/Z)

\section{Author details}

${ }^{1}$ Electronics and Nanoscale Engineering, James Watt School of Engineering, University of Glasgow, Glasgow G12 8QQ, UK. ${ }^{2}$ Beatson West of Scotland Cancer Centre, Glasgow G12 OYN, UK. ${ }^{3}$ School of Science and Technology, Nottingham Trent University, Nottingham NG11 8NF, UK. ${ }^{4}$ School of Sport, Exercise \& Health Sciences, Loughborough University, Loughborough LE11 3TU, UK. ${ }^{5}$ Glasgow Polyomics, College of Medical, Veterinary and Life Sciences, University of Glasgow, Glasgow G61 1BD, UK. ${ }^{6}$ Institute of Cancer Sciences, Beatson West of Scotland Cancer Centre, University of Glasgow, Glasgow G12 OYN, UK. Institute of Infection, Immunity and Inflammation, University of Glasgow, Glasgow G12 8TA, UK

\section{Author contributions}

V.F.A. and D.R.S.C. conceived and designed the research. C.G., M.A.A.-R., and J.B. designed the integrated chip. J.G., Y.D.S., and B.C.C. provided technical support for the development and testing of the microfluidics, and S.B.P. helped with the planning of the experiments. C.A. and M.A.A.-R. designed the reader. C.A. provided support for V.F.A. in the development of the experimental setup. V.F.A. developed methods and protocols. M.M., T.R.J.E., and R.J. provided the clinical samples, supported by the Glasgow Experimental Cancer Medicine Centre (ECMC), funded by Cancer Research UK and the Chief Scientist's Office, Scotland. S.B.P. and C.H. provided technical support to V.F.A. during the calibration and the clinical study. D.J.C. and L.M.H. performed independent sample testing. V.F.A. acquired the data. V.F.A. analyzed the data. R.D. trained and characterized the classifier. V.F.A. performed the experiments for simultaneous testing. R.J. and M.P.B. supervised the clinical study. V.F.A. and D.R.S.C. wrote the manuscript. All authors reviewed the manuscript.

\section{Conflict of interest}

The authors declare no competing interests.

Supplementary information The online version contains supplementary material available at https://doi.org/10.1038/s41378-021-00243-4.

Received: 31 July 2020 Revised: 5 November 2020 Accepted: 15 December 2020

Published online: 05 March 2021

\section{References}

1. Ahmad, A. S., Ormiston-Smith, N. \& Sasieni, P. D. Trends in the lifetime risk of developing cancer in Great Britain: comparison of risk for those born from 1930 to 1960. Br. J. Cancer 112, 943-947 (2015).

2. WHO. World Health Organization. https://www.who.int/ (accessed Jan. 17, 2020).

3. Prostate Cancer UK. https://prostatecanceruk.org/ (accessed Mar. 11, 2020).

4. Public Health England. "Chapter 2: Major Causes of Death and How They Have Changed - GOV.UK" https:/www.gov.uk/government/publications/healthprofile-for-england/chapter-2-major-causes-of-death-and-how-they-havechanged (accessed Feb. 04, 2021).

5. Smittenaar, C. R., Petersen, K. A., Stewart, K. \& Moitt, N. Cancer incidence and mortality projections in the UK until 2035. Br. J. Cancer 115, 1147-1155 (2016).

6. Rawla, P. Epidemiology of prostate cancer. Rev. World J. Oncol. 10, 63-89 (2019).

7. Culp, M. B. B., Soerjomataram, I., Efstathiou, J. A., Bray, F. \& Jemal, A. Recent global patterns in prostate cancer incidence and mortality rates. Eur. Urol. 77, 38-52 (2020).

8. Etzioni, R. et al. The case for early detection. Nat. Rev. Cancer 3, 243-252 (2003).

9. Hayes, B., Murphy, C., Crawley, A. \& O'Kennedy, R. Developments in point-ofcare diagnostic technology for cancer detection. Diagnostics 8, 39 (2018).

10. Hugosson, J. et al. A 16-yr follow-up of the european randomized study of screening for prostate cancer (Figure presented.). Eur. Urol. 76, 43-51 (2019).

11. Walsh, P. C. Operating characteristics of prostate-specific antigen in men with an initial PSA level of $3.0 \mathrm{Ng} / \mathrm{Ml}$ or lower: commentary. J. Urol. 175, 562-563 (2006).

12. Gómez-Cebrián, N. et al. Metabolomics contributions to the discovery of prostate cancer biomarkers. Metabolites $\mathbf{9}, 48$ (2019).

13. $\mathrm{Ma}, \mathrm{X}$. et al. The cost implications of prostate cancer screening in the Medicare population. Cancer 120, 96-102 (2014).

14. Lilienfeld, S. O., Alliger, G. \& Mitchell, K. Why integrity testing remains controversial. Am. Psychol. 50, 457-458 (1995).

15. Fridhammar, A., Axelsson, U., Persson, U., Bjartell, A. \& Borrebaeck, C. A. K. The value of a new diagnostic test for prostate cancer: a cost-utility analysis in early stage of development. PharmacoEconomics https://doi.org/10.1007/s41669020-00226-7 (2020).

16. Meyer, A. R. \& Gorin, M. A. First point-of-care PSA test for prostate cancer detection. Nat. Rev. Urol. 16, 332-333 (2019).

17. Damborska, D. et al. Nanomaterial-based biosensors for detection of prostate specific antigen. Microchim. Acta 184, 3049-3067 (2017).

18. Zhang, T., He, Y., Wei, J. \& Que, L. Nanostructured optical microchips for cancer biomarker detection. Biosens. Bioelectron. 38, 382-388 (2012).

19. Salisbury, T. B. \& Arthur, S. The regulation and function of the L-type amino acid transporter 1 (LAT1) in cancer. Int. J. Mol. Sci. 19, 2373 (2018).

20. Huang, J. et al. Serum metabolomic profiling of prostate cancer risk in the prostate, lung, colorectal, and ovarian cancer screening trial. Br. J. Cancer $\mathbf{1 1 5}$ 1087-1095 (2016). 
21. Johansson, M. et al. One-carbon metabolism and prostate cancer risk: Prospective investigation of seven circulating B vitamins and metabolites. Cancer Epidemiol. Biomark. Prev. 18, 1538-1543 (2009).

22. Glunde, K., Penet, M. F., Jiang, L., Jacobs, M. A. \& Bhujwalla, Z. M. Choline metabolism-based molecular diagnosis of cancer: an update. Expert Rev. Mol. Diagn. 15, 735-747 (2015)

23. Trock, B. J. Application of metabolomics to prostate cancer. Urologic Oncol. Semin. Original Investig. 29, 572-581 (2011).

24. Kelly, R. S., Heiden, M. G. V., Giovannucci, E. \& Mucci, L. A. Metabolomic biomarkers of prostate cancer: prediction, diagnosis, progression, prognosis, and recurrence. Cancer Epidemiol. Biomark. Prev. 25, 887-906 (2016).

25. Sreekumar, A. et al. Metabolomic profiles delineate potential role for sarcosine in prostate cancer progression. Nature 457, 910-914 (2009).

26. Locasale, J. W. Serine, glycine and one-carbon units: cancer metabolism in full circle. Nat. Rev. Cancer 13, 572-583 (2013).

27. Soliman, L. C., Hui, Y., Hewavitharana, A. K. \& Chen, D. D. Y. Monitoring potential prostate cancer biomarkers in urine by capillary electrophoresistandem mass spectrometry. J. Chromatogr. A 1267, 162-169 (2012).

28. Kobayashi, M., Kurokawa, S. \& Tokue, A. Intraindividual variation in total and percent free prostate-specific antigen levels in prostate cancer suspects. Urol. Int. 74, 198-202 (2005).

29. Pagkalos, l., Herrero, P., Toumazou, C., Georgiou, P. \& Member, S. Bio-Inspired glucose control in diabetes based on an analogue implementation of a cell model. IEEE Trans. Biomed. Circuits Syst. 8, 186-195 (2014).

30. Xu, G., Abbott, J. \& Ham, D. Optimization of CMOS-ISFET-based biomolecular sensing: analysis and demonstration in DNA detection. IEEE Trans. Electron Devices 63, 3249-3256 (2016).

31. Shekar, S. et al. A miniaturized multi-clamp CMOS amplifier for intracellular neural recording. Nat. Electron. 2, 343-350 (2019).

32. Rothberg, J. M. et al. An integrated semiconductor device enabling nonoptical genome sequencing. Nature 475, 348-352 (2011)

33. Patil, S. B. et al. An integrated portable system for single chip simultaneous measurement of multiple disease associated metabolites. Biosens. Bioelectron. 122, 88-94 (2018).

34. Huang, Y. \& Mason, A. J. Lab-on-CMOS integration of microfluidics and electrochemical sensors. Lab Chip 13, 3929-3934 (2013).

35. Ghafar-Zadeh, E., Sawan, M. \& Therriault, D. Novel direct-write CMOS-based laboratory-on-chip: design, assembly and experimental results. Sens. Actuat. A Phys. 134, 27-36 (2007).

36. Welch, D. \& Christen, J. B. Seamless integration of CMOS and microfluidics using flip chip bonding. J. Micromech. Microeng. 23, 035009 (2013).

37. Zhang, B., Dong, Q., Korman, C. E., Li, Z. \& Zaghloul, M. E. Flexible packaging of solid-state integrated circuit chips with elastomeric microfluidics. Sci. Rep. 3, 1-8 (2013).

38. Chin, C. D., Linder, V. \& Sia, S. K. Commercialization of microfluidic point-of-care diagnostic devices. Lab Chip 12, 2118-2134 (2012).

39. Gubala, V., Harris, L. F., Ricco, A. J., Tan, M. X. \& Williams, D. E. Point of care diagnostics: status and future. Anal. Chem. 84, 487-515 (2012).

40. Kimura, J., Kawana, Y. \& Kuriyama, T. An immobilized enzyme membrane fabrication method using an ink jet nozzle. Biosensors 4, 41-52 (1989).

41. Kalyanaraman, B. Teaching the basics of cancer metabolism: developing antitumor strategies by exploiting the differences between normal and cancer cell metabolism. Redox Biol. 12, 833-842 (2017).

42. Vazquez, A. et al. Cancer metabolism at a glance. J. Cell Sci. 129, 3367-3373 (2016).

43. Shen, J., Yan, L., Liu, S., Ambrosone, C. B. \& Zhao, H. Plasma metabolomic profiles in breast cancer patients and healthy controls: by race and tumor receptor subtypes. Transl. Oncol. 6, 757-765 (2013).

44. Wishart, D. S. Emerging applications of metabolomics in drug discovery and precision medicine. Nat. Rev. Drug Discov. 15, 473-484 (2016).

45. Zhang, T. et al. Discrimination between malignant and benign ovarian tumors by plasma metabolomic profiling using ultra performance liquid chromatography/mass spectrometry. Clin. Chim. Acta 413, 861-868 (2012).
46. Giskeødegård, G. F. et al. Metabolic markers in blood can separate prostate cancer from benign prostatic hyperplasia. Br. J. Cancer 113, 1712-1719 (2015).

47. Koochekpour, S. et al. Serum glutamate levels correlate with gleason score and glutamate blockade decreases proliferation, migration, and invasion and induces apoptosis in prostate cancer cells. Clin. Cancer Res. 18, 5888-5901 (2012).

48. Jóźwiak, P., Forma, E., Bryś, M. \& Krześlak, A. O-GlcNAcylation and metabolic reprograming in cancer. Front. Endocrinol. 5, 145 (2014).

49. Struys, E. A., Heijboer, A. C., Van Moorselaar, J., Jakobs, C. \& Blankenstein, M. A. Serum sarcosine is not a marker for prostate cancer. Ann. Clin. Biochem. 47, 282 (2010).

50. Bohm, L. et al. Plasma sarcosine does not distinguish early and advanced stages of prostate cancer. South Afr. Med. J. 102, 677-679 (2012).

51. Cheah, B. C. et al. An integrated circuit for chip-based analysis of enzyme kinetics and metabolite quantification. IEEE Trans. Biomed. Circuits Syst. 10, 721-730 (2016).

52. Swinehart, D. F. The Beer-Lambert law. J. Chem. Educ. 39, 333 (1962).

53. Annese, V. F. et al. The Multicorder: A Handheld Multimodal Metabolomics-onCMOS Sensing Platform. In Proc. 2019 8th International Workshop on Advances in Sensors and Interfaces, IWASI 2019. https://doi.org/10.1109/ IWASI.2019.8791347 (2019).

54. Al-Rawhani, M. A. et al. Multimodal integrated sensor platform for rapid biomarker detection. IEEE Trans. Biomed. Eng. https:/doi.org/10.1109/ TBME.2019.2919192 (2019).

55. Trantidou, T., Elani, Y., Parsons, E. \& Ces, O. Hydrophilic surface modification of pdms for droplet microfluidics using a simple, quick, and robust method via pva deposition. Microsystems Nanoeng. 3, 16091 (2017).

56. Enzyme Database - BRENDA. https://www.brenda-enzymes.org/index.php (accessed Jan. 22, 2020).

57. Long, G. L. \& Winefordner, J. D. Limit of detection: a closer look at the IUPAC definition. Anal. Chem. 55, 712A-724A (1983).

58. Chen, T. et al. Random forest in clinical metabolomics for phenotypic discrimination and biomarker selection. Evidence-Based Complement. Altern. Med. 2013, 298183 (2013).

59. Liaw, A. \& Wiener, M. Classification and regression by randomForest. R News $\mathbf{2}$ 18-22 (2003).

60. Kuhn, M. Building predictive models in $\mathrm{R}$ using the caret package. J. Stat. Softw. 28, 1-26 (2008)

61. Thompson, I. M. et al. Operating characteristics of prostate-specific antigen in men with an initial PSA level of $3.0 \mathrm{ng} / \mathrm{mL}$ or lower. JAMA $\mathbf{2 9 4}, 66-70$ (2005).

62. Crawford, E. D. \& Abrahamsson, P. A. PSA-based screening for prostate cancer: how does it compare with other cancer screening tests? Eur. Urol. 54, 262-273 (2008).

63. Cancer Research UK. https://www.cancerresearchuk.org/ (accessed Apr. 17, 2020).

64. Reddy, B. et al. Point-of-care sensors for the management of sepsis. Nat. Biomed. Eng. 2, 640-648 (2018).

65. Cánovas, R., Cuartero, M. \& Crespo, G. A. Modern creatinine (Bio)sensing: challenges of point-of-care platforms. Biosens. Bioelectron. 130, 110-124 (2019).

66. Hand, M. Access to timely and optimal care of patients with acute coronary syndromes-Community planning considerations: a report by the National Heart Attack Alert Program. J. Thromb. Thrombolysis 6, 19-46 (1998).

67. Accarino, $C$. et al. Noise characteristics with CMOS sensor array scaling. Meas. J. Int. Meas. 152, 107325 (2020).

68. Aquilani, R. et al. The relationship between plasma amino acids and circulating albumin and haemoglobin in postabsorptive stroke patients. PLOS ONE 14, 1-21 (2019).

69. Pitkin R. M., Allen L. H. \& Bailey L. B., Dietary Reference Intakes for Thiamin, Riboflavin, Niacin, Vitamin B6, Folate, Vitamin B12, Pantothenic Acid, Biotin, and Choline. (National Academies Press, 1998). 